\title{
AMPK activation by ASP4132 inhibits non-small cell lung cancer cell growth
}

\author{
Ying-chen Xia', Jian-hua Zha', Yong-Hua Sang ${ }^{2}$, Hui Yin', Guo-qiu Xu', Jie Zhen³, Yan Zhang ${ }^{4}$ and Ben-tong Yu (D)
}

\begin{abstract}
Activation of adenosine monophosphate-activated protein kinase (AMPK) is able to produce significant anti-non-small cell lung cancer (NSCLC) cell activity. ASP4132 is an orally active and highly effective AMPK activator. The current study tested its activity against NSCLC cells. In primary NSCLC cells and established cell lines (A549 and NCI-H1944) ASP4132 potently inhibited cell growth, proliferation and cell cycle progression as well as cell migration and invasion. Robust apoptosis activation was detected in ASP4132-treated NSCLC cells. Furthermore, ASP4132 treatment in NSCLC cells induced programmed necrosis, causing mitochondrial p53-cyclophilin D (CyPD)-adenine nucleotide translocase 1 (ANT1) association, mitochondrial depolarization and medium lactate dehydrogenase release. In NSCLC cells ASP4132 activated AMPK signaling, induced AMPKa1-ACC phosphorylation and increased AMPK activity. Furthermore, AMPK downstream events, including mTORC1 inhibition, receptor tyrosine kinases (PDGFRa and EGFR) degradation, Akt inhibition and autophagy induction, were detected in ASP4132-treated NSCLC cells. Importantly, AMPK inactivation by AMPKa1 shRNA, knockout (using CRISPR/Cas9 strategy) or dominant negative mutation (T172A) almost reversed ASP4132-induced anti-NSCLC cell activity. Conversely, a constitutively active AMPKa1 (T172D) mimicked and abolished ASP4132-induced actions in NSCLC cells. In vivo, oral administration of a single dose of ASP4132 largely inhibited NSCLC xenograft growth in SCID mice. AMPK activation, mTORC1 inhibition and EGFR-PDGFRa degradation as well as Akt inhibition and autophagy induction were detected in ASP4132-treated NSCLC xenograft tumor tissues. Together, activation of AMPK by ASP4132 potently inhibits NSCLC cell growth in vitro and in vivo.
\end{abstract}

\section{Introduction}

Lung cancer accounts for over $13 \%$ of all new cancer diagnoses ${ }^{1,2}$. In the United States alone, an estimated of 228,820 adults will be diagnosed with lung cancer each year $^{1,2}$. Of which non-small cell lung cancer (NSCLC) constitutes close to $85 \%$ of all lung cancers ${ }^{1,2}$. NSCLC main subtypes include adenocarcinoma, squamous cell carcinoma, and large cell carcinoma ${ }^{1,2}$. Due to the lack of specific symptoms and ineffectiveness of current

\footnotetext{
Correspondence: Yan Zhang (zlnkunshan@163.com) or Ben-

tong Yu (yubentongnc@hotmail.com)

'Department of Thoracic Surgery, The First Affiliated Hospital of Nanchang

University, Nanchang, China

2Department of Thoracic Surgery, The Second affiliated Hospital of Soochow

University, Suzhou, China

Full list of author information is available at the end of the article

These authors contributed equally: Ying-chen Xia, Jian-hua Zha,

Yong-Hua Sang

Edited by A. Finazzi-Agrò
}

therapies, NSCLC prognosis is far from satisfactory. The five-year overall survival (OS) for stage I NSCLC is 47\%, stage II is $30 \%$, stage III is close to $10 \%$, and stage IV is less than $1 \%^{1,2}$.

Adenosine monophosphate-activated protein kinase (AMPK) is a highly-conserved eukaryotic protein kinase, playing an essential role in cellular energy homeostasis ${ }^{3-5}$. With the cellular energy decreasing, activated AMPK is able to promote glucose and fatty acid uptake and oxidation $^{3-5}$. AMPK is composed of $\alpha, \beta$, and $\gamma$ subunits $^{3-5}$. AMPK can be activated with AMP binding or by phosphorylation at $\alpha$ subunit's Thr-172 residue ${ }^{6}$. One of primary AMPK kinases is the tumor suppressor protein kinase $\mathrm{LKB1}^{7}$. Exonic and whole LKB1 gene deletions are presented in Peutz-Jeghers syndrome with a strong tendency of developing cancer $^{8,9}$. In addition, somatic mutations and inactivation of LKB1 are associated with lung cancers $^{10}$. Gill et al., observed that loss of

\section{(c) The Author(s) 2021}

(c) (i) Open Access This article is licensed under a Creative Commons Attribution 4.0 International License, which permits use, sharing, adaptation, distribution and reproduction cc) in any medium or format, as long as you give appropriate credit to the original author(s) and the source, provide a link to the Creative Commons license, and indicate if changes were made. The images or other third party material in this article are included in the article's Creative Commons license, unless indicated otherwise in a credit line to the material. If material is not included in the article's Creative Commons license and your intended use is not permitted by statutory regulation or exceeds the permitted use, you will need to obtain permission directly from the copyright holder. To view a copy of this license, visit http://creativecommons.org/licenses/by/4.0/. 
heterozygosity or homozygous deletion of LKB1 gene was occurred in the majority of NSCLCs ${ }^{10}$. Interestingly, Hui et al., have shown that AMPK $\alpha 1$ expression levels are significantly higher in NSCLC tumor tissues ${ }^{11}$. These results implied that AMPK cascade could be an important therapeutic target of NSCLC.

Activation of AMPK could lead to metabolic tumor suppression due to energy metabolism regulation, metabolic checkpoint enforcement and growth inhibition ${ }^{12}$. AMPK activators are potential therapeutic candidates for cancer treatment ${ }^{12-16}$. Indeed, forced activation of AMPK signaling cascade, using pharmacological or genetic strategies, could exert potent anti-NSCLC cell activity. Chen et al., have shown that chrysin activated AMPK signaling cascade to cause growth inhibition and apoptosis in A549 NSCLC cells ${ }^{17}$. Conversely, AMPK inhibition or silencing largely attenuated chrysin-induced anti-A549 cell activity ${ }^{17}$. Wei et al. reported that cordycepin, by activating AMPK signaling, induced apoptosis in drug-resistance NSCLC cells $^{18}$. Moreover, AMPK activation by metformin inhibited NSCLC cell growth and enhanced radiation sensitivity ${ }^{19}$. Furthermore, AMPK cascade activation by Circular RNA circHIPK3 induced autophagic death in NSCLC cells ${ }^{20}$.

Although activation of AMPK could exert profound anti-NSCLC cell activity ${ }^{17-20}$. The traditional AMPK activators, including AICAR and metformin, were often utilized at extreme high concentrations (at $\mathrm{mM}$ concentrations in vitro) $)^{14,21,22}$. Furthermore, these known AMPK activators could induce AMPK-independent toxicities ${ }^{14,21,22}$. Recent studies have developed ASP4132 as an orally active and potent AMPK activator with an EC50 of $18 \mathrm{nM}^{23,24}$. This novel AMPK activator has displayed potent and selective cell growth inhibitory activity against breast cancer cell lines in vitro ${ }^{24}$. Furthermore, oral administration of ASP4132 has shown potent in vivo anti-tumor efficacy in a MDA-MB-453 xenograft mouse model $^{24}$. Metabolic stability and in vivo animal pharmacokinetics (PK) profiles of ASP4132 are favorable ${ }^{24}$. Its aqueous solubility at gastrointestinal $\mathrm{pH}$ is also acceptable ${ }^{23,24}$. We therefore hypothesized that activation of AMPK by ASP4132 could possibly exert potent antiNSCLC cell activity.

\section{Materials and methods}

\section{Chemicals and reagents}

ASP4132 was purchased from MCE China (Beijing, China). ASP4132 powder was first dissolved in DMSO to $100 \mathrm{mM}$ stock solution. For in vitro studies, ASP4132 stock solution was further dissolved in PBS before adding to cell culture medium. For in vivo studies, ASP4132 stock solution was dissolved in 40\% PEG400-5\% Tween- 80 saline solution and applied by oral gavage. Antibodies of p-AMPK $\alpha 1$ (Thr172, \#2531), AMPK $\alpha 1$ (\#2532), acetyl-CoA Carboxylase (ACC, \#3662), p-ACC
(Ser79, \#3661), p-Akt (Thr308, \#5110), Akt1/2 (\#2967), GAPDH (glyceraldehyde-3-phosphate dehydrogenase, \#5174), p70 S6 Kinase (S6K1 \#9202), p-S6K1 (Thr389, \#9205), p-S6 Ribosomal Protein (Ser235/236, \#2211), EGFR (\#2232), PDGFR $\alpha$ (\#4547), cleaved caspase antibody sampler Kit (\#9929), Erk1/2 (\#4695), and $\beta$-Tubulin (\#2146) were purchased from Cell Signaling Technologies (Beverly, MA). The autophagy antibody kit was purchased from Cell Signaling Technologies (\#4445) as well. The anti-adenine nucleotide translocase 1 (ANT1) antibody (ab102032) was purchased from Abcam China (Shanghai, China). Antibodies for cyclophilin-D (CyPD, sc-137136) and p53 (sc-126) were provided by Santa Cruz Biotech (Santa Cruz, CA). All reagents for cell culture, including fetal bovine serum (FBS) and antibiotics, were provided by Hyclone (Logan, UT). From Sigma-Aldrich Chemicals Co. (St. Louis, Mo) puromycin, polybrene, z-DEVD-fmk, zVAD-fmk and all other chemicals were obtained. Shanghai Genechem Co. (Shanghai, China) synthesized and verified all primers, sequences, constructs and virus, unless otherwise mentioned. JC-1, EdU, DAPI, TUNEL and CellROX fluorescence dyes, Annexin V, propidium iodide (PI) and PCR reagents were purchased from Thermo-Fisher Invitrogen (Shanghai, China).

\section{Cell culture}

NSCLC cell lines, NCI-H1944 and A549, were purchased from Shanghai Institute of Biochemistry and Cell Biology (Shanghai, China). Cells were cultured in DMEM together with $10 \%$ FBS. Primary human NSCLC cells, derived from three written-informed consent NSCLC patients (pNSCLC$1 /-2 /-3$ ), as well as BEAS-2B lung epithelial cells and primary lung epithelial cells, were provided by Dr. Jiang ${ }^{25,26}$, and cells were cultured as described previously ${ }^{25,26}$. No patients were treated with any additional therapies before surgery. The protocols of using human cells were approved by the Ethics Committee of Nanchang University (NCUBR099), in according to Declaration of Helsinki. All cells utilized in this study were subjected to mycoplasma and microbial contamination examination every two months. Authentication by STR profiling, population doubling time, and cell morphology were routinely checked to verify their genotypes.

\section{Cell viability}

NSCLC cells or lung epithelial cells were seeded into 96-well plates (at $3 \times 10^{5}$ cells $/ \mathrm{mL}$ ). Following the applied treatment CCK-8 was added to each well. CCK-8 absorbance (optical density, OD) was detected by a plate reader at the test wavelength of $550 \mathrm{~nm}$.

\section{"Transwell" assays}

Following the applied treatment, NSCLC cells were trypsinized, re-suspended into serum-free medium and 
added on the upper surfaces of Transwell chambers (BD Biosciences, Shanghai, China). The lower chambers were filled with complete medium with $10 \%$ FBS. After $24 \mathrm{~h}$ of migration, the migrated cells in the lower chambers were fixed, stained and counted. Invasion assays were conducted using the same protocol except the "Transwell" inserts were pre-coated with Matrigel (Sigma).

\section{EdU staining}

NSCLC cells were initially seeded into 12 -well plates at $3 \times 10^{4}$ cells per well and were allowed to adhere overnight and subjected to treatments accordingly. Afterwards, cells were washed, fixed and stained with EdU for $2 \mathrm{~h}$. Cell nuclei were co-stained with DAPI. For each condition at least 1200 nuclei from five random microcopy views $(1 \times 100)$ were counted to calculate EdUpositive nuclei ratio (EdU/DAPI $\times 100 \%$ ).

\section{Clonogenic assays}

NSCLC cells were initially seeded into six-well plates (10,000 cells per well) and maintained at the ASP4132containing complete medium. After ten days colonies were fixed, stained and counted.

\section{Cell cycle studies}

NSCLC cells were seeded at $0.4 \times 10^{6}$ cells per well and subjected to applied treatment. Afterwards, cells were washed in PBS, trypsinized, centrifuged and resuspended in $1 \mathrm{~mL}$ of $95 \%$ ethanol and stored at $-20{ }^{\circ} \mathrm{C}$ for $24 \mathrm{~h}$. Cells were then centrifuged and resuspended in $1 \mathrm{~mL}$ of propidium iodide (PI) staining solution, and eventually were tested under a FACS-calibur flow cytometry (Beckman-Coulter, Shanghai, China). Data analyses were performed via an Expo32 ADC v1.1c software.

\section{Annexin-V assay}

NSCLC cells were seeded at $1 \times 10^{6}$ cells per $\mathrm{mL}$ and subjected to applied treatment. Cells were then washed, resuspended and fixed. Afterwards, cells were incubated with PI $(10 \mu \mathrm{g} / \mathrm{mL})$ and Annexin V $(10 \mu \mathrm{g} / \mathrm{mL})$, and were examined by the FACS-calibur flow cytometry (BeckmanCoulter). Annexin V-positive cells were gated as apoptotic cells and its ratio was recorded.

\section{Apoptotic nuclei assay}

NSCLC cells were seeded onto a glass cover-slip and subjected to applied treatment. Cells were then rinsed in PBS and co-stained with Hoechst-33342 and TUNEL dyes. Apoptotic nuclei presented with condensed DNA and fragmented nuclei staining (certain apoptotic nuclei were positive with TUNEL staining). For each condition at least 1200 nuclei from five random microcopy views $(1 \times 100)$ were counted to calculate apoptotic nuclei ratio.

\section{Western blotting}

Cells and tissues were incubated with the lysis buffer (as described ${ }^{18}$ ). From each treatment protein lysates (40 $\mu$ g per lane) were resolved by 10-12\% SDS-PAGE gels and transferred to PVDF membranes (Millipore, Shanghai, China). Membranes were incubated sequentially in PBST containing $10 \%$ non-fat milk, followed by incubation with primary antibody (overnight at $4{ }^{\circ} \mathrm{C}$ ) and secondary antibody ( $2 \mathrm{~h}$ at room temperature). An ECL Western Blotting Substrate Kit (Abcam, Shanghai, China) was utilized to visualize the targeted protein band. Image J software from NIH website was utilized for data quantification.

\section{Quantitative real time-PCR (qPCR) assay}

Total cellular RNA was extracted via TRIzol reagents (Thermo-Fisher Invitrogen) and was reverse-transcripted. The ABI Prism 7900 Fast Real-Time PCR system was utilized for qPCR assays. The product melting temperature was calculated. Quantification of targeted mRNAs was through the $2^{-\triangle \Delta C t}$ method, using GAPDH as an internal control. The mRNA primers for EGFR, PDGFR and GAPDH were from Dr. Chen at Jiangsu University ${ }^{27}$.

\section{Caspase-3 activity}

Following treatment the hypotonic cell lysis buffer ${ }^{18}$ was utilized to extract cytosolic proteins. The protein lysates ( $20 \mu \mathrm{g}$ per treatment) were added to the caspase assay buffer ${ }^{18}$ containing the caspase- 3 substrate (Calbiochem, Darmstadt, Germany). After $2 \mathrm{~h}$ incubation, released 7 amido-4-(trifluoromethyl)coumarin (AFC) was quantified through a Fluoroskan system (Thermo-Labsystems, Helsinki, Finland) ${ }^{18}$.

\section{Mitochondrial immunoprecipitation (Mito-IP)}

The detailed procedures of Mito-IP were reported early $^{28,29}$. In brief, mitochondrial lysates were obtained from NSCLC cells with applied treatment ${ }^{30,31}$. Lysates (500 $\mu \mathrm{g}$ per condition) were pre-cleared and incubated with anti-cyclophilin-D (CypD) antibody (Santa Cruz Biotech). CypD-immunoprecipitated proteins, adenine nucleotide translocator-1 (ANT1) and p53, were captured and tested by Western blotting.

\section{Mitochondrial depolarization}

JC-1 is able to accumulate in mitochondria as monomers in cells undergoing mitochondrial membrane potential (MMP) reduction. With mitochondria depolarization JC-1 will then emit green fluorescence $(490 \mathrm{~nm})^{32}$. The detailed protocols of JC-1 assaying of mitochondrial depolarization were described early ${ }^{33}$. JC-1 fluorescence images, merging both the green fluorescence channel and the red fluorescencence channel, were presented. 


\section{AMPKa1 shRNA}

NSCLC cells were seeded into six-well plates at 50\% confluence and treated with AMPK $\alpha 1$ shRNA lentiviral particles (Sigma-Aldrich, $20 \mu \mathrm{L}$ per well). NSCLC cells were further cultured for $48 \mathrm{~h}$. Afterwards, cells were cultured in complete medium containing puromycin $(2.5 \mu \mathrm{g} / \mathrm{mL})$. After six passages AMPK $\alpha 1$ expression in the stable cells was examined by Western blotting assays.

\section{AMPKa1 KO}

The CRISPR/Cas9 AMPK $\alpha 1-K O$ construct was provided by Dr. Pan at Shanghai Jiao Tong University School of Medicine ${ }^{34}$ and was transduced to pNSCLC-1 cells by Lipofectamine 3000. Transfected cells were distributed to 192-well plates and subjected to AMPK $\alpha 1-\mathrm{KO}$ screen. The achieved single cells were further selected by puromycin to achieve stable AMPK $1-\mathrm{KO}$ cells.

\section{AMPK mutation}

The constitutively active AMPK $\alpha 1$ (T172D, caAMPK $\alpha 1$ ) adenoviral vector and the dominant negative AMPK $\alpha 1$ (T172A, dnAMPK $\alpha 1)$ adenoviral vector, both containing GFP and puromycin selection gene, were provided by Dr. Wang at Soochow University ${ }^{35}$. NSCLC cells were seeded into six-well plates at $50 \%$ confluence and transfected with caAMPK $\alpha 1$ vector or $\operatorname{dnAMPK} \alpha 1$ vector using Lipofectamine 3000 (Thermo-Fisher Invitrogen). Transfected cells with GFP were sorted by FACS and distributed into 192-well plates. Single stable cells were further selected by puromycin-containing medium. The empty vector was transfected by Lipofectamine 3000 (Invitrogen, Carlsbad, CA) to control cells.

\section{AMPK activity assay}

Total cellular lysates were initially incubated with an anti-AMPK $\alpha 1$ antibody (Santa Cruz Biotech). AMPK activity was measured by using AMP- $\left[\gamma^{-32} \mathrm{P}\right]$ ATP mixture and AMPK substrate SAMS (HMRSAMSGLHLVKRR) peptide. To stop the reaction the phosphocellulose paper was included in the kinase assay buffer. The AMPK radioactivity was examined by a scintillation counter.

\section{Light chain 3B (LC3B) staining}

As reported ${ }^{36,37}$, NSCLC cells with applied treatment were fixed and incubated with anti-LC3B antibody (red fluorescence protein/RFP-conjugated, Genechem, Shanghai, China). LC3B RFP fluorescence puncta in the autophagic cells was visualized under a Leica microscope.

\section{NSCLC mouse xenograft assay}

Five-week-old severe combined immunodeficient (SCID) mice (half male half female, $18.5-19.5 \mathrm{~g}$ in weights) were maintained under Animal Facility of Soochow University Medical School (Suzhou, China). Each mice was grafted with $3 \times 10^{6}$ pNSCLC1 cells through s.c. injection to the right flank. Within three weeks when tumor volume reached $100 \mathrm{~mm}^{3}$ (labeled as "Day-0") animals were randomly assigned into two groups, receiving either vehicle control or the applied ASP4132 administration (ten mice per group, $n=10$ ). Tumor dimensions were measured by calliper every six days and volume was estimated as per: $V=$ length $\times$ width $\times$ height $\times 0.5236$. At experimental Day-7 and Day-14, one tumor of each group was extracted via surgery. Tumors were cut into small pieces and lysed in tissue lysis buffer (Biyuntian, Wuxi, China). Protein lysates were tested by Western blotting. All animal experiments were approved by Animal Ethics Board of Nanchang University (ID: NCUBR015).

\section{Statistical analysis}

All in vitro experiments in this study were repeated five times with similar results obtained. Data were presented as mean \pm standard deviation (SD, $n=5$ ). Error bars stand for five replicated wells/dishes for each in vitro experiment. One-way ANOVA (plus Student-Newman-Keuls post hoc test) was carried out to determine statistical significance between multiple groups (SPSS23.0, SPSS Co. Chicago, CA). A two-tailed Student's $t$-test (Excel 2007) was utilized when comparing difference between two specific groups. Values of $P<0.05$ were considered as statistically different.

\section{Results}

ASP4132 treatment exerts potent anti-NSCLC cell activity

The primary human NSCLC cells, pNSCLC-1 (as reported early ${ }^{25,26}$ ), were cultured in FBS (10\%)-containing complete medium and treated with graduallyincreased concentrations of ASP4132 $(0.1-3.0 \mu \mathrm{M})$. CCK- 8 assay was performed to test cell viability. Results in Fig. 1A showed that ASP4132 decreased pNSCLC-1 cell viability in a concentration-dependent manner. ASP4132, at $0.3-3.0 \mu \mathrm{M}$, was significant in inhibiting cell viability (Fig. 1A), but was ineffective at $0.1 \mu \mathrm{M}$ (Fig. 1A). The AMPK activator also showed a time-dependent response in inhibiting pNSCLC-1 cell viability, as it required at least $48 \mathrm{~h}$ to exert a significant effect (Fig. 1A). In Fig. 1B the colony formation assay results displayed that ASP4132 $(0.3-3.0 \mu \mathrm{M})$ significantly decreased the number of viable pNSCLC-1 cell colonies, further supporting its antisurvival activity. Testing cell proliferation, using EdU incorporation assay, demonstrated that ASP4132 dosedependently decreased the ratio of EdU-positive nuclei in pNSCLC-1 cells (Fig. 1C). ASP4132 at $0.1 \mu \mathrm{M}$ again failed to significantly inhibit colony formation (Fig. 1B) and EdU incorporation (Fig. 1C). Since $1 \mu \mathrm{M}$ of ASP4132 potently inhibited cell viability (Fig. 1A), colony formation (Fig. 1B) and EdU incorporation (Fig. 1C), this concentration was selected for the following studies. 




The PI-FACS assay was carried out to examine cell cycle progression. In ASP4132-treated pNSCLC-1 cells, G1phase cells were increased, while S-phase cells were reduced (Fig. 1D). These results indicated that the AMPK activator disrupted cell cycle progression and induced G1$S$ arrest in pNSCLC-1 cells (Fig. 1D). "Transwell" assays were utilized to test cell migration. Results showed that ASP4132 $(1 \mu \mathrm{M})$ treatment significantly decreased the number of migrated pNSCLC-1 cells (Fig. 1E). In addition, pNSCLC-1 cell invasion, tested by "Matrigel Transwell" assays, was inhibited by ASP4132 treatment as well (Fig. 1F). Notably, for the "Transwell" assays pNSCLC-1 cells were treated with ASP4132 for only $24 \mathrm{~h}$ with no significant viability reduction detected (Fig. 1A).

Next the potential effect of ASP4132 was tested in other NSCLC cells. The primary NSCLC cells that were derived from two other patients, pNSCLC-2 and pNSCLC-3, as well as immortalized NSCLC cell lines (A549 and NCIH1944), were cultured. With ASP4132 $(1 \mu \mathrm{M})$ treatment, CCK-8 viability was significantly decreased in the primary and established NSCLC cells (Fig. 1G). Furthermore, in the NSCLC cells the AMPK activator inhibited cell 
proliferation and migration, which were tested by EdU incorporation (results quantified in Fig. 1H) and "Transwell" (results quantified in Fig. 1I) assays, respectively. In primary lung epithelial cells ("pEpi") and established BEAS-2B epithelial cells ${ }^{38}$, ASP4132 $(1 \mu \mathrm{M}, 72 \mathrm{~h})$ treatment failed to significantly inhibit cell viability (Fig. 1J), indicating a cancer cell specific effect by the AMPK activator. Together, in NSCLC cells ASP4132 potently inhibited cell viability, proliferation and cell cycle progression as well as cell migration and invasion.

\section{ASP4132 induces apoptosis activation in NSCLC cells}

Forced activation of AMPK, through genetic or pharmacological strategies, was able to provoke cancer cell apoptosis ${ }^{17,39,40}$. In Fig. 1 we showed that ASP4132 induced proliferation inhibition and cell cycle arrest, we next tested its effect on cell apoptosis. As shown ASP4132 $(1 \mu \mathrm{M})$ treatment significantly increased caspase-3 activity in pNSCLC-1 cells (Fig. 2A). The AMPK activator induced robust cleavages of caspase-3, caspase-9 and poly (ADP-ribose) polymerase (PARP) in pNSCLC-1 cells (Fig. 2B). Single strand DNA (ssDNA) accumulation, indicating DNA breaks, was detected in ASP4132-treated cells (Fig. 2C). Importantly, the ratio of apoptotic nuclei was dramatically increased following ASP4132 treatment in pNSCLC-1 cells (Fig. 2D). The apoptotic nuclei showed condensed or fragmented nuclear (Hoechst-33342) staining (characteristic nuclei were labeled with yellow stars, Fig. 2D), of which some were also TUNEL-positive (Fig. 2D). To further confirm apoptosis activation, FACS assay results showed that ASP4132 significantly increased the number of Annexin $\mathrm{V}$-positive cells (Fig. 2E). Increased trypan blue staining further confirmed the cytotoxic effect of the AMPK activator in pNSCLC-1 cells (Fig. 2F).

In other primary (pNSCLC-2 and pNSCLC-3) and established (A549 and NCI-H1944 lines) NSCLC cells, ASP4132 treatment $(1 \mu \mathrm{M}, 48 \mathrm{~h})$ significantly increased caspase-3 activity (Fig. 2G) and the apoptotic nuclei ratio (Fig. 2H). In contrast, no significant apoptosis activation was detected in primary ("pEpi") and established (BEAS2B) lung epithelial cells with the same ASP4132 treatment (Fig. 2I), further supporting cancer cell specific response by the AMPK activator. These results clearly showed that ASP4132 activated apoptosis in NSCLC cells.

\section{ASP4132 activates programmed necrosis cascade in NSCLC cells}

To block apoptosis activation two caspase inhibitors, including the caspase-3 inhibitor z-DEVD-fmk and the pan caspase inhibitor z-VAD-fmk, were utilized. By recording apoptotic nuclei ratio, Fig. 3A, we showed that z-DEVD-fmk and z-VAD-fmk blocked ASP4132-induced apoptosis activation in pNSCLC-1 cells (Fig. 3A).
However, the two only inhibited, but did not reverse, ASP4132-induced viability (CCK-8 OD) reduction (Fig. 3B) and cell death (Trypan blue ratio increase, Fig. 3C). These results suggested that ASP4132-induced cytotoxicity in NSCLC cells was not solely due to apoptosis activation.

Besides apoptosis, cancer cells can undergo programmed necrosis when facing various stimuli and anticancer agents ${ }^{41,42}$. Programmed necrosis is a mitochondria-dependent active cell death process ${ }^{41,42}$. Using the mitochondrial immunoprecipitation ("MitoIP") assay, we found that ASP4132 induced cyclophilin-D (CypD) association with p53 and adenine nucleotide translocator-1 (ANT1) in the mitochondria of pNSCLC-1 cells (Fig. 3D, "Mito-IP: CyPD"). It is a key initial step of programmed necrosis cascade activation ${ }^{41,43,44}$. Mitochondrial expression of CyPD, p53 and ANT1 was unchanged with ASP4132 treatment (Fig. 3D, "Input").

Furthermore in ASP4132-treated pNSCLC-1 cells, profound mitochondrial depolarization was detected (Fig. $3 \mathrm{E})$, evidenced by JC-1 green monomers accumulation in mitochondria ${ }^{45}$. These events were followed by LDH release to the medium (Fig. 3F), a characteristic marker of cell membrane rupture and cell necrosis. Therefore, ASP4132 induced mitochondrial CyPD-p53-ANT1 association (Fig. 3D), mitochondrial depolarization (Fig. 3E) and medium LDH release (Fig. 3F), suggesting activation of the programmed necrosis cascade (see studies testing the same cascade ${ }^{43,46-49}$ ).

To block programmed necrosis, we utilized a CyPD inhibitor cyclosporin A (CsA $)^{41,44}$. Alternatively, CyPD shRNA lentiviral particles were added to pNSCLC-1 cells, and via puromycin selection stable cells were established (sh-CyPD cells). CyPD protein expression was silenced in sh-CyPD pNSCLC-1 cells (Fig. 3G). As shown CsA or CyPD shRNA inhibited ASP4132-induced viability (CCK$8 \mathrm{OD}$ ) reduction and cell death (Fig. $3 \mathrm{H}$ ), suggesting that the programmed necrosis should also participate in ASP4132-induced anti-NSCLC cell activity. However, ASP4132-induced apoptosis activation in pNSCLC-1 cells was not affected by CsA or CyPD shRNA (Supplementary Fig. S1A). The two caspase inhibitors, z-DEVD-fmk and zVAD-fmk, failed to attenuate ASP4132-induced medium LDH release (the necrosis indicator) in pNSCLC-1 cells (Supplementary Fig. S1B). In pNSCLC-2 cells ASP4132 treatment similarly induced mitochondrial depolarization (JC-1 green monomers intensity, Fig. 3I) and cell necrosis (medium LDH release, Fig. 3J). Collectively, these results confirmed that ASP4132 activated programmed necrosis cascade in NSCLC cells.

\section{ASP4132 activates AMPK signaling in NSCLC cells}

ASP4132 is a novel AMPK activator ${ }^{23,24}$, we therefore tested its effect on AMPK signaling in NSCLC cells. 


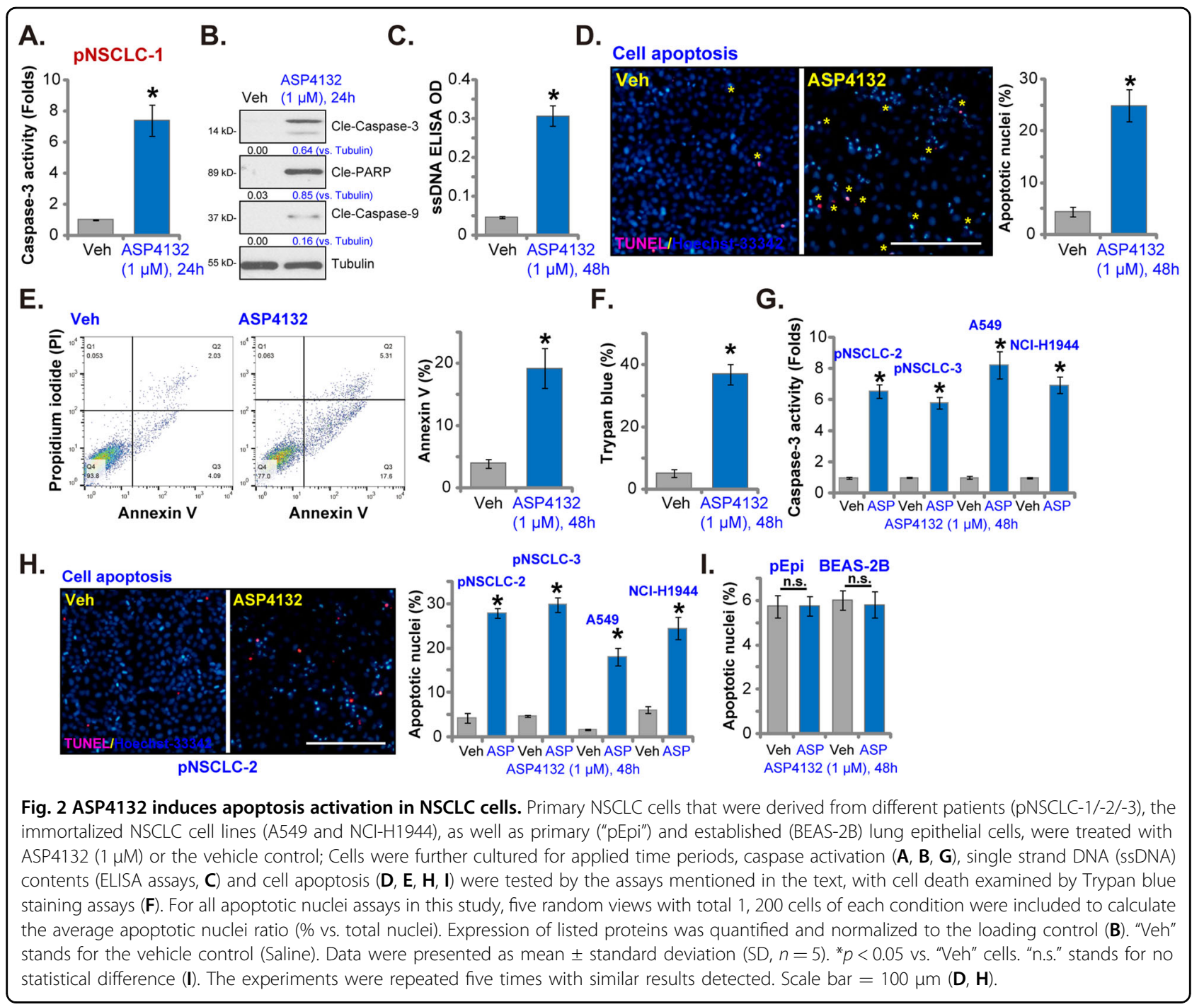

Western blotting assay results, Fig. 4A, demonstrated that ASP4132 $(1 \mu \mathrm{M})$ induced robust phosphorylation of AMPK $\alpha 1$ (Thr-172) and ACC (Ser79) in pNSCLC-1 and pNSCLC-2 cells. AMPK $\alpha 1$ and ACC protein expression was unchanged (Fig. 4A). In addition, the AMPK activity increased over twenty folds in ASP4132-treated NSCLC cells (Fig. 4B). These results indicated that ASP4132 activated AMPK signaling in NSCLC cells.

Activated AMPK is able to exert anti-cancer cell activity by regulating its targets, including mammalian target of rapamycin (mTOR) complex 1 (mTORC1) inhibition ${ }^{50-}$ 52 , autophagy induction ${ }^{52-54}$ and degradation of various oncogenes ${ }^{27,55}$. We showed that phosphorylation of S6K1, the indicators of mTORC1 activation, was almost completely blocked by ASP4132 in pNSCLC-1 and pNSCLC-2 cells (Fig. 4C). A previous study by Chen et al., has demonstrated that AMPK activation is able to induce lysosomal translocation and degradation of PDGFR and
$\mathrm{EGFR}^{27}$. We here found that protein levels of PDGFR $\alpha$ and EGFR were significantly decreased in ASP4132treated NSCLC cells as well (Fig. 4D). PDGFR $\alpha$ and EGFR mRNA levels were however unchanged (Fig. 4D). Akt phosphorylation at Thr-308, the key downstream signal molecular of PDGFR $\alpha$ and EGFR, was also inhibited (Fig. 4D).

In addition, in pNSCLC-1 and pNSCLC-2 cells ASP4132 treatment induced LC3B-I conversion to LC3BII (Fig. 4E), the characteristic marker of autophagy induction $^{56}$. Furthermore, intense LC3B-II RFP (red fluorescence protein) puncta were accumulated in the cytosol of ASP4132-treated pNSCLC-1 and pNSCLC-2 cells (Fig. 4F), confirming autophagy induction. Collectively, these results showed that ASP4132 activated AMPK signaling, causing mTORC1 inhibition, PDGFR $\alpha$ EGFR protein degradation, Akt inactivation, and autophagy induction in NSCLC cells. 




\section{AMPK activation mediates ASP4132-induced anti-NSCLC cell activity}

To test AMPK activation is the primary reason of ASP4132-induced anti-NSCLC cell activity, lentiviral AMPK $\alpha 1$ shRNA was transduced to pNSCLC-1 cells. Stable cells were established following selection by puromycin: shAMPK $\alpha 1$ cells. In addition a CRISPR/Cas9AMPK $\alpha 1-K O$ construct was transfected to pNSCLC-1 cells. Single stable cells were established following GFP sorting plus puromycin selection: koAMPK $\alpha 1$ cells. As shown, AMPK $\alpha 1$ protein expression was depleted in both shAMPK $\alpha 1$ cells and koAMPK $\alpha 1$ cells (Fig. 5A). ASP4132-induced AMPK activation, or AMPK $\alpha 1-A C C$ phosphorylation, was blocked in AMPK $\alpha 1$-silenced and
AMPK $\alpha 1-K O$ pNSCLC-1 cells (Fig. 5A). Significantly, ASP4132-induced viability (CCK-8 OD) reduction (Fig. $5 \mathrm{~B}$ ), cell apoptosis (increase of apoptotic nuclei ratio, Fig. $5 \mathrm{C}$ ) and death (Fig. 5D) were reversed in shAMPK $\alpha 1$ cells and koAMPK 1 cells. Therefore, AMPK inhibition reversed ASP4132-induced anti-NSCLC cell activity, suggesting that AMPK activation was required for ASP4132-induced anti-NSCLC cell activity.

Next, a dominant negative AMPK $\alpha 1$ (T172A, dnAMPK $\alpha 1^{27,57}$ ) was transduced to pNSCLC-1 cells, and stable cells were established: dnAMPK $\alpha 1$ cells. As shown the construct, dnAMPK $\alpha 1$, largely inhibited ASP4132induced AMPK $\alpha 1-\mathrm{ACC}$ phosphorylation in pNSCLC-1 cells (Fig. 5E). Consequently, ASP4132-induced viability 


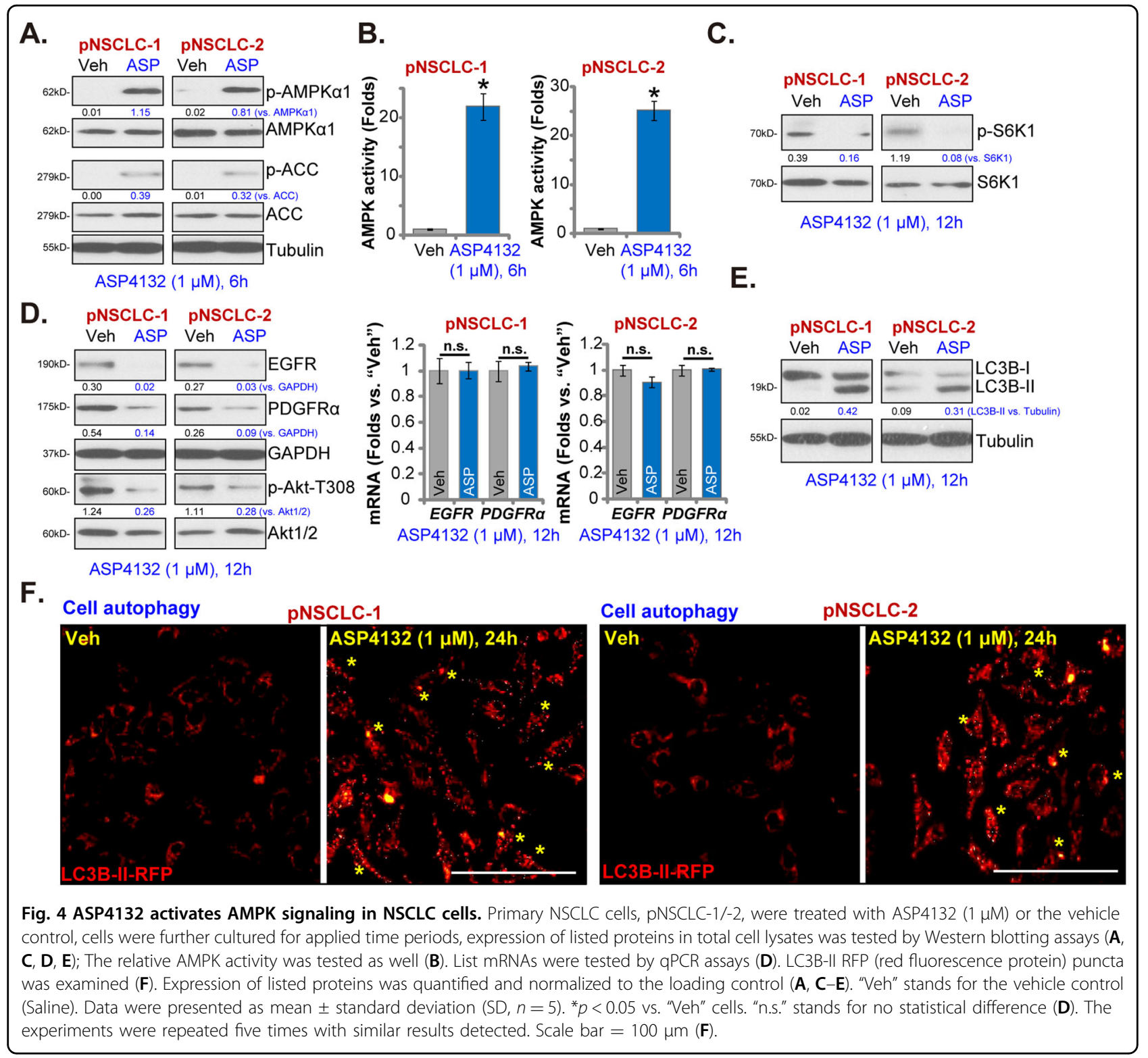

reduction (Fig. 5F), cell apoptosis (Fig. 5G) and death (Fig. $5 \mathrm{H})$ were significantly attenuated in dnAMPK $\alpha 1$ cells (Fig. 5F-H). These results further supported that AMPK activation mediated ASP4132-induced anti-NSCLC cell activity.

To further support our hypothesis, a constitutively active AMPK $\alpha 1$ (T172D, caAMPK $\alpha 1^{35,58,59}$ ) was stably transfected to pNSCLC-1 cells, resulting in robust increase of AMPK $\alpha 1-\mathrm{ACC}$ phosphorylation (Fig. 5I). Mimicking ASP4132-induced actions, caAMPK $\alpha 1$ induced significant anti-NSCLC cell activity, causing viability reduction (Fig. 5J), cell apoptosis (Fig. 5K) and death (Fig. 5L). Importantly, in caAMPK $\alpha 1$-expressing pNSCLC-1 cells adding ASP4132 failed to further increase AMPK 1 1-ACC phosphorylation (Fig. 5I). Neither did it produce further anti-pNSCLC-1 cell activity (Fig. 5J-L). Thus, ASP4132 was invalid on cell behaviors in caAMPK $\alpha 1$ NSCLC cells, further supporting that AMPK activation is the primary reason of ASP4132-induced antiNSCLC cell activity.

\section{Oral administration of ASP4132 inhibits NSCLC xenograft growth in SCID mice}

Next we tested whether ASP4132 could inhibit NSCLC cell growth in vivo. As described, three weeks after pNSCLC-1 cells injection to SCID mice flanks, tumor xenografts were established ("Day-0"). Mice were then randomly assigned into two groups, receiving ASP4132 or vehicle administration. By recording tumor volumes we found that oral administration of a single dose of 


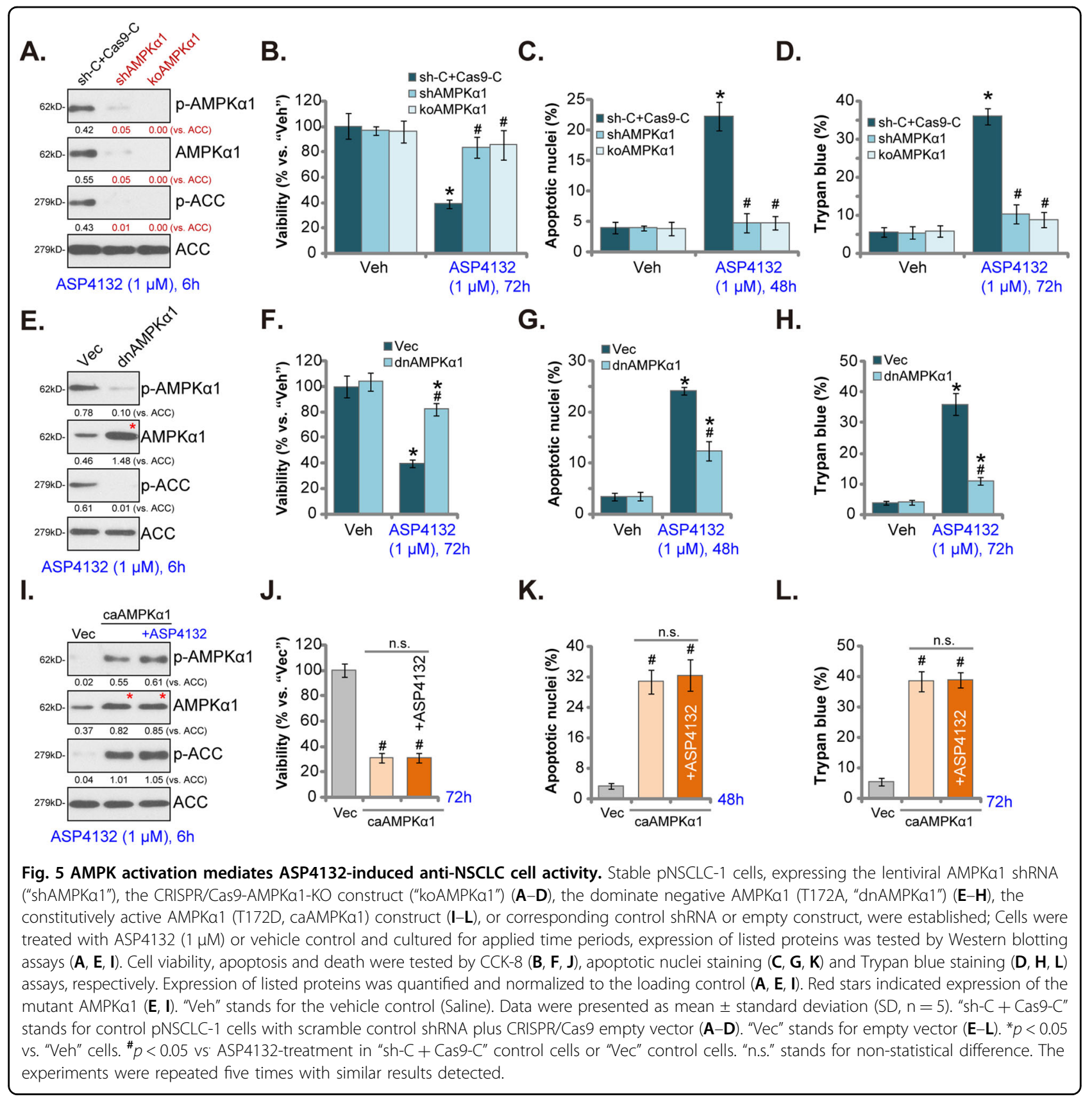

ASP4132 ( $5 \mathrm{mg} / \mathrm{kg}$ body weight, for 21 days) largely inhibited NSCLC xenograft growth in SCID mice (Fig. 6A). This concentration was based on the recommendation from the supplier. Volumes of NSCLC xenografts with ASP4132 administration were significantly lower than those of vehicle control xenografts (Fig. 6A). We next applied a formula to calculate the estimated daily tumor growth: (Tumor volume at Day-42-Tumor volume at Day-0)/42. Results showed that ASP4132 oral administration potently suppressed NSCLC xenograft growth in SCID mice (Fig. 6B). At Day-42 all tumors of the two groups were separated through surgery and weighted individually. Results in Fig. 6C demonstrated that ASP4132-treated NSCLC xenografts were dramatically lighter than the control tumors. Importantly, the mice body weights were not significantly different between the ASP4132 group and vehicle control group (Fig. 6D), indicating that mice should be well-tolerated to ASP4132 treatment regimen, and we did not detect any apparent toxicities.

At experimental Day-7 and Day-14, $4 \mathrm{~h}$ after initial ASP4132/vehicle administration, one tumor of each 


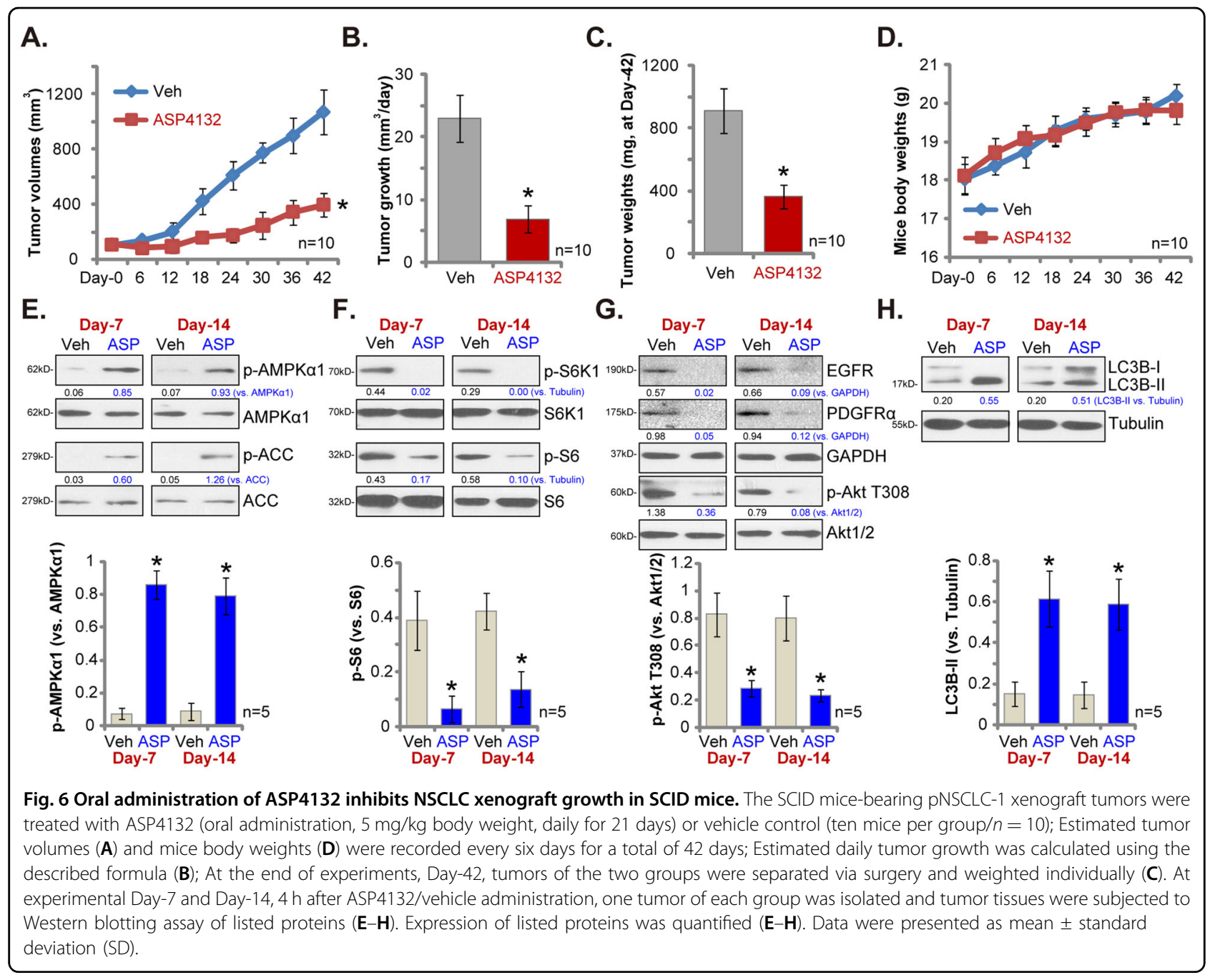

group was separated via surgery. Each tumor was cut into five small pieces and lysed separately in the tissue lysis buffer. Western blotting assays were carried out to test signaling proteins. As shown p-AMPK 11 and p-ACC levels were significantly elevated in ASP4132-stimualted NSCLC xenograft tissues (Fig. 6E), indicating AMPK signaling activation. To support mTORC1 inhibition in vivo, we found that p-S6K1 and p-S6 were largely inhibited in NSCLC xenograft tissues with ASP4132 administration (Fig. 6F). Furthermore, ASP4132 induced EGFR and PDGFR $\alpha$ protein degradation as well as Akt inhibition in NSCLC xenograft tissues (Fig. 6G). Moreover, LC3B-I to LC3B-II conversion in ASP4132-treated NSCLC xenograft tissues suggested autophagy activation in vivo (Fig. 6H). Quantitative analyses integrating all blotting data testing five samples per tumor $(n=5)$ confirmed that AMPK $\alpha 1$ phosphorylation, p-S6 inhibition, pAkt inhibition and LC3B-II conversion were significant in tumor tissues with ASP4132 administration (Fig. 6E-H, lower panels). Therefore, in consistent with in vitro signaling findings, ASP4132 administration induced AMPK activation, mTORC1 inhibition, RTKs (EGFR and PDGFR $\alpha$ ) degradation and downstream Akt inactivation, as well as autophagy induction in NSCLC xenograft tissues.

\section{Discussion}

Lung cancer is still one of the leading causes of cancerassociated mortalities, as it accounts for almost one-fifth of all cancer death worldwide ${ }^{1,2,60}$. Of all lung cancer, $85 \%$ of them are $\mathrm{NSCLC}^{60}$. One primary reason of poor prognosis of NSCLC is the overwhelming resistance to current therapies ${ }^{61-64}$. Therefore, the development of novel and more efficacious therapeutics against this devastating disease is urgent ${ }^{61-64}$.

Several other AMPK activators were reported to exert potent anti-NSCLC cell activity, including cordycepin ${ }^{18,65}$, polyphyllin $\mathrm{I}^{66}$, baicalin ${ }^{67}$ and metformin ${ }^{19,68}$. These 
tested AMPK activators, howerever, required high concentrations (over $10-100 \mu \mathrm{M}$ in vitro) to induce significant anticancer activities ${ }^{18,65-67}$. Poor water solubility and potential off-target cytotoxicity might limit its application in vivo ${ }^{18,65-67}$. Here in cultured primary NSCLC cells (pNSCLC-1/-2/-3) and established cell lines (A549 and NCI-H1944) a low concentration of ASP4132 $(1 \mu \mathrm{M})$ can potently inhibited cell growth, viability and proliferation as well as cell migration and invasion (Fig. 1). In addition, robust cell cycle arrest and apoptosis activation were detected in ASP4132-treated NSCLC cells (Figs. 13). In vivo, oral administration of a single dose of ASP4132 largely inhibited NSCLC xenograft growth in SCID mice without inducing apparent toxicity (Fig. 6). Therefore, ASP4132 is a novel and potent anti-NSCLC cell agent.

Due to mutation, amplification, deletion, methylation and post-translational modifications, mTOR overactivation in NSCLC is essential for tumorigenesis, cancer progression and therapy resistance, and is often associated with poor prognosis ${ }^{69,70}$. Wang et al., found that SH2B1, a member of the SH2-domain containing family, activated mTOR signaling to promote NSCLC cell proliferation ${ }^{71}$. Kumar et al., found that PI3K-mTOR inhibition by antroquinonol inhibited NSCLC cell proliferation ${ }^{72}$. mTOR-driven cancer progression can be inhibited by activation of $\mathrm{AMPK}^{73-75}$. Activated AMPK is able to phosphorylate and activate TSC2 (Tuberous sclerosis protein 2) to block mTORC1 activation ${ }^{50}$. In addition AMPK phosphorylates and in-activates Raptor (regulatory associated protein of mTOR) to further inhibit mTORC1 ${ }^{76}$. In the present study we showed that ASP4132 potently inhibited mTORC1 activation and blocked S6K1-S6 phosphorylation in NSCLC cells (Fig. 4). Furthermore, mTORC1 inactivation was detected in ASP4132-treated NSCLC xenograft tumor tissues (Fig. 6). Therefore, mTOR inactivation might be one important reason to explain ASP4132-induced anti-NSCLC cell activity.

Autophagy activation could play a positive and negative role in NSCLC progression ${ }^{77}$. The general idea is that the persistent activation of autophagy shall be able to induce autophagic cell death in NSCLC cells ${ }^{77,78}$. Activated AMPK directly associates with and phosphorylates the autophagyinitiating kinase Ulk1, which is the most important upstream component of the autophagy machinery ${ }^{52,53,79}$. Moreover, activated AMPK phosphorylates and activates TSC2 to suppress mTORC1 activation, leading to autophagy induction ${ }^{50,51}$. Jang et al., found that AMPK activation is required for autophagosome maturation and lysosomal fusion $^{80}$. Here we found that AMPK activation by ASP4132 induced autophagy activation, causing LC3B-I to LC3B-II conversion and LC3B-GFP puncta formation in NSCLC cells (Fig. 4). LC3B-I to LC3B-II conversion was detected in
NSCLC xenograft tumor tissues with ASP4132 administration as well (Fig. 6). These results implied that autophagy activation could actively participate in ASP4132induced anti-NSCLC activity.

Besides apoptosis, programmed necrosis is another form of active cell death that occurs in cancer cells facing different stimuli. In colorectal cancer cells PF-543, a novel sphingosine kinase 1 (SphK1) inhibitor, provoked programmed necrosis by inducing MMP reduction and mitochondrial p53-CyPD complexation ${ }^{49}$. Qin et al., reported that salinomycin-induced anti-glioma cell activity was associated with programmed necrosis cascade activation ${ }^{41}$. Zhang et al., found that berberine-induced cytotoxicity in prostate cancer cells was mainly due to activation of CyPD-dependent programmed necrosis pathway $^{43}$. In the present study, we found that ASP4132 induced programmed necrosis (together with apoptosis) in NSCLC cells, causing mitochondrial p53-CyPD-ANT1 association, mitochondrial depolarization and medium LDH release (Fig. 3). Significantly, CyPD shRNA or inhibition (CsA) alleviated ASP4132-induced cytotoxicity in NSCLC cells (Fig. 3). Therefore, concurrent activation of two independent cell death cascades, apoptosis and programmed necrosis, could explain the superior antiNSCLC cell activity by this novel AMPK activator. The detailed mechanisms of programmed necrosis activation by the AMPK activator warrant further studies.

Chen et al., have demonstrated that forced activation of AMPK could induce degradation of multiple RTKs (PDGFR $\alpha$, PDGFR $\beta$ and EGFR) by promoting their lysosomal translocation ${ }^{27}$. mRNA expression of these RTKs was however unchanged ${ }^{27}$. Here we displayed that ASP4132 treatment similarly induced PDGFR $\alpha$-EGFR protein degradation and inhibited downstream Akt activation in NSCLC cells and xenograft tumor tissues (Figs. 4 and 6). Considering the critical role of RTK-Akt activation in NSCLC progression ${ }^{69,70}$, targeting this cascade by ASP4132 might be another reason of its superior antiNSCLC cell activity.

In the current study the findings from in vitro assays and animal studies cannot be directly translated to humans. The safety and the anti-NSCLC efficiency of ASP4132 should be further tested. The respective contribution of apoptosis, programmed necrosis and autophagy to ASP4132-induced NSCLC cell death, as well as their relationships, warrant further investigations.

\section{Conclusion}

Activation of AMPK by ASP4132 potently inhibits NSCLC cell growth in vitro and in vivo.

\footnotetext{
Acknowledgements

We thank Dr. Zhang at Suzhou University for study design and manuscript proofreading.
} 


\section{Author details}

'Department of Thoracic Surgery, The First Affiliated Hospital of Nanchang University, Nanchang, China. ${ }^{2}$ Department of Thoracic Surgery, The Second affiliated Hospital of Soochow University, Suzhou, China. ${ }^{3}$ Department of Thoracic Surgery, Qidong People's Hospital, Qidong, China. ${ }^{4}$ Department of Radiotherapy and Oncology, Affiliated Kunshan Hospital of Jiangsu University, Kunshan, China

\section{Author contributions}

All listed authors designed the study, performed the experiments and the statistical analysis, and wrote the manuscript and revise it. All authors have read the manuscript and approved the final version.

\section{Funding}

This study is supported by the National Natural Science Foundation of China (81660391, 81773192 and 82072712), the Natural Science Foundation of Jiangxi Province (20202BABL206088) and by Special Fund for Postgraduate Innovation of Jiangxi Province (Grant No. YC2020-B056), Health Personnel Training Project of Suzhou (GSWS2020113) and by Research Project of Jiangsu Province health committee (Z2019054).

\section{Conflict of interest}

The authors declare no competing interests.

\section{Ethics Statement}

This study was approved by the Ethics Committee of Nanchang University.

\section{Publisher's note}

Springer Nature remains neutral with regard to jurisdictional claims in published maps and institutional affiliations.

Supplementary information The online version contains supplementary material available at https://doi.org/10.1038/s41419-021-03655-2.

Received: 12 December 2020 Revised: 17 March 2021 Accepted: 18 March 2021

Published online: 06 April 2021

\section{References}

1. Siegel, R. L., Miller, K. D. \& Jemal, A. Cancer statistics, 2020. CA Cancer J. Clin. 70, 7-30 (2020)

2. Siegel, R. L., Miller, K. D. \& Jemal, A. Cancer statistics, 2019. CA Cancer J. Clin. 69, 7-34 (2019).

3. Liu, Y. J. \& Chern, Y. AMPK-mediated regulation of neuronal metabolism and function in brain diseases. J. Neurogenet. 29, 50-58 (2015).

4. Mihaylova, M. M. \& Shaw, R. J. The AMPK signalling pathway coordinates cell growth, autophagy and metabolism. Nat. Cell Biol. 13, 1016-1023 (2011).

5. Luo, Z., Saha, A. K., Xiang, X. \& Ruderman, N. B. AMPK, the metabolic syndrome and cancer. Trends Pharm. Sci. 26, 69-76 (2005).

6. Hardie, D. G., Ross, F. A. \& Hawley, S. A. AMPK: a nutrient and energy sensor that maintains energy homeostasis. Nat. Rev. Mol. Cell Biol. 13, 251-262 (2012).

7. Shackelford, D. B. \& Shaw, R. J. The LKB1-AMPK pathway: metabolism and growth control in tumour suppression. Nat. Rev. Cancer 9, 563-575 (2009).

8. Hearle, N. et al. Frequency and spectrum of cancers in the Peutz-Jeghers syndrome. Clin. Cancer Res. 12, 3209-3215 (2006).

9. Lim, W. et al. Further observations on LKB1/STK11 status and cancer risk in Peutz-Jeghers syndrome. Br. J. Cancer 89, 308-313 (2003).

10. Gill, R. K. et al. Frequent homozygous deletion of the LKB1/STK11 gene in nonsmall cell lung cancer. Oncogene 30, 3784-3791 (2011).

11. Hui, G. D. et al. AMP-activated protein kinase alpha1 serves a carcinogenic role via regulation of vascular endothelial growth factor expression in patients with non-small cell lung cancer. Oncol. Lett. 17, 4329-4334 (2019).

12. Li, W., Saud, S. M., Young, M. R., Chen, G. \& Hua, B. Targeting AMPK for cancer prevention and treatment. Oncotarget 6, 7365-7378 (2015).

13. Choi, Y. K. \& Park, K. G. Metabolic roles of AMPK and metformin in cancer cells. Mol. Cells 36, 279-287 (2013).
14. Faubert, B., Vincent, E. E., Poffenberger, M. C. \& Jones, R. G. The AMP-activated protein kinase (AMPK) and cancer: many faces of a metabolic regulator. Cancer Lett. 356, 165-170 (2015).

15. Kim, I. \& He, Y. Y. Targeting the AMP-activated protein kinase for cance prevention and therapy. Front Oncol. 3, 175 (2013).

16. Vallianou, N. G., Evangelopoulos, A. \& Kazazis, C. Metformin and cancer. Rev. Diabet. Stud. 10, 228-235 (2013).

17. Shao, J. J. et al. AMP-activated protein kinase (AMPK) activation is involved in chrysin-induced growth inhibition and apoptosis in cultured A549 lung cancer cells. Biochem. Biophys. Res. Commun. 423, 448-453 (2012).

18. Wei, C. et al. Cordycepin inhibits drug-resistance non-small cell lung cancer progression by activating AMPK signaling pathway. Pharm. Res. 144, 79-89 (2019).

19. Storozhuk, Y. et al. Metformin inhibits growth and enhances radiation response of non-small cell lung cancer (NSCLC) through ATM and AMPK. Br. J. Cancer 108, 2021-2032 (2013).

20. Chen, X. et al. Circular RNA circHIPK3 modulates autophagy via MIR124-3pSTAT3-PRKAAVAMPKalpha signaling in STK11 mutant lung cancer. Autophagy 16, 659-671 (2020)

21. Hardie, D. G., Ross, F. A. \& Hawley, S. A. AMP-activated protein kinase: a target for drugs both ancient and modern. Chem. Biol. 19, 1222-1236 (2012).

22. Kim, J., Yang, G., Kim, Y., Kim, J. \& Ha, J. AMPK activators: mechanisms of action and physiological activities. Exp. Mol. Med. 48, e224 (2016).

23. Kuramoto, K. et al. Novel indirect AMP-activated protein kinase activators: identification of a second-generation clinical candidate with improved physicochemical properties and reduced hERG inhibitory activity. Chem. Pharm. Bull. 68, 452-465 (2020).

24. Kuramoto, $\mathrm{K}$. et al. Development of a potent and orally active activator of adenosine monophosphate-activated protein kinase (AMPK), ASP4132, as a clinical candidate for the treatment of human cancer. Bioorg. Med Chem. $\mathbf{2 8}$ 115307 (2020).

25. $Y u, H .$, Chen, Y. \& Jiang, P. Circular RNA HIPK3 exerts oncogenic properties through suppression of miR-124 in lung cancer. Biochem. Biophys. Res Commun. 506, 455-462 (2018).

26. Zhang, B., Lu, H. Y., Xia, Y. H., Jiang, A. G. \& LV, Y. X. Long non-coding RNA EPIC1 promotes human lung cancer cell growth. Biochem. Biophys. Res Commun. 503, 1342-1348 (2018).

27. Chen, M. B. et al. Itraconazole-induced inhibition on human esophagea cancer cell growth requires AMPK activation. Mol. Cancer Ther. 17, 1229-1239 (2018).

28. Shi, X., Liu, H. Y., Li, S. P. \& Xu, H. B. Keratinocyte growth factor protects endometrial cells from oxygen glucose deprivation/re-oxygenation via activating Nif2 signaling. Biochem. Biophys. Res Commun. 501, 178-185 (2018).

29. Tang, X. F. et al. Ginseng Rh2 protects endometrial cells from oxygen glucose deprivation/re-oxygenation. Oncotarget 8, 105703-105713 (2017).

30. Zhou, L. N., Li, P., Cai, S., Li, G. \& Liu, F. Ninjurin2 overexpression promotes glioma cell growth. Aging (Albany NY). 11, 11136-11147 (2019).

31. Li, G. et al. Ninjurin 2 overexpression promotes human colorectal cancer cell growth in vitro and in vivo. Aging (Albany NY). 11, 8526-8541 (2019).

32. Brooks, M. M., Neelam, S., Fudala, R., Gryczynski, I. \& Cammarata, P. R. Lenticular mitoprotection. Part A: monitoring mitochondrial depolarization with JC-1 and artifactual fluorescence by the glycogen synthase kinase-3beta inhibitor, SB216763. Mol. Vis. 19, 1406-1412 (2013).

33. Liang, J. et al. PGK1 depletion activates Nrf2 signaling to protect human osteoblasts from dexamethasone. Cell Death Dis. 10, 888 (2019).

34. Xue, J. et al. Lnc-THOR silencing inhibits human glioma cell survival by activating MAGEA6-AMPK signaling. Cell Death Dis. 10, 866 (2019).

35. Guo, S. et al. Activating AMP-activated protein kinase by an alpha1 selective activator compound 13 attenuates dexamethasone-induced osteoblast cell death. Biochem. Biophys. Res Commun. 471, 545-552 (2016).

36. Zhu, L. Q. et al. Salinomycin activates AMP-activated protein kinase-dependent autophagy in cultured osteoblastoma cells: a negative regulator against cell apoptosis. PLoS ONE 8, e84175 (2013).

37. Zhen, Y. F. et al. Identification of DNA-PKcs as a primary resistance factor of salinomycin in osteosarcoma cells. Oncotarget 7, 79417-79427 (2016).

38. Yang, X., Lei, P., Huang, Y., Zhang, Z. \& Zhang, Y. MicroRNA-133b inhibits the migration and invasion of non small cell lung cancer cells via targeting FSCN1. Oncol. Lett. 12, 3619-3625 (2016).

39. Kim, Y. M., Hwang, J. T., Kwak, D. W., Lee, Y. K. \& Park, O. J. Involvement of AMPK signaling cascade in capsaicin-induced apoptosis of HT-29 colon cancer cells. Ann. N. Y. Acad. Sci. 1095, 496-503 (2007). 
40. Kang, M. R. et al. Widdrol induces apoptosis via activation of AMP-activated protein kinase in colon cancer cells. Oncol. Rep. 27, 1407-1412 (2012).

41. Qin, L. S., Jia, P. F., Zhang, Z. Q. \& Zhang, S. M. ROS-p53-cyclophilin-D signaling mediates salinomycin-induced glioma cell necrosis. J. Exp. Clin. Cancer Res. 34, 57 (2015).

42. Zhou, C. et al. Icaritin activates JNK-dependent MPTP necrosis pathway in colorectal cancer cells. Tumour Biol. 37, 3135-3144 (2016)

43. Zhang, L. Y., Wu, Y. L., Gao, X. H. \& Guo, F. Mitochondrial protein cyclophilin-Dmediated programmed necrosis attributes to berberine-induced cytotoxicity in cultured prostate cancer cells. Biochem. Biophys. Res Commun. 450, 697-703 (2014).

44. Guo, F., Liu, S. Q., Gao, X. H. \& Zhang, L. Y. AlCAR induces AMPK-independent programmed necrosis in prostate cancer cells. Biochem. Biophys. Res Commun. 474, 277-283 (2016).

45. Cossarizza, A., Baccarani-Contri, M., Kalashnikova, G. \& Franceschi, C. A new method for the cytofluorimetric analysis of mitochondrial membrane potential using the J-aggregate forming lipophilic cation 5,5',6,6'-tetrachloro1,1',3,3'-tetraethylbenzimidazolcarbocyanine iodide (JC-1). Biochem. Biophys. Res Commun. 197, 40-45 (1993).

46. Feoktistova, M. \& Leverkus, M. Programmed necrosis and necroptosis signalling. FEBS J. 282, 19-31 (2015).

47. Xie, J., Li, Q., Ding, X. \& Gao, Y. GSK1059615 kills head and neck squamous cell carcinoma cells possibly via activating mitochondrial programmed necrosis pathway. Oncotarget 8, 50814-50823 (2017).

48. Sun, Q., Shen, X., Wang, P., Ma, J. \& Sha, W. Targeting cyclophilin-D by miR1281 protects human macrophages from Mycobacterium tuberculosisinduced programmed necrosis and apoptosis. Aging 11, 12661-12673 (2019).

49. Ju, T., Gao, D. \& Fang, Z. Y. Targeting colorectal cancer cells by a novel sphingosine kinase 1 inhibitor PF-543. Biochem. Biophys. Res Commun. 470, 728-734 (2016)

50. Inoki, K., Zhu, T. \& Guan, K. L. TSC2 mediates cellular energy response to control cell growth and survival. Cell 115, 577-590 (2003).

51. Inoki, K. et al. TSC2 integrates Wnt and energy signals via a coordinated phosphorylation by AMPK and GSK3 to regulate cell growth. Cell 126, 955-968 (2006).

52. Kim, J., Kundu, M., Viollet, B. \& Guan, K. L. AMPK and mTOR regulate autophagy through direct phosphorylation of Ulk1. Nat. Cell Biol. 13, 132-141 (2011).

53. Egan, D. F. et al. Phosphorylation of ULK1 (hATG1) by AMP-activated protein kinase connects energy sensing to mitophagy. Science 331, 456-461 (2011).

54. Huo, H. Z. et al. AMP-activated protein kinase (AMPK)/Ulk1-dependent autophagic pathway contributes to C6 ceramide-induced cytotoxic effects in cultured colorectal cancer HT-29 cells. Mol. Cell Biochem. 378, 171-181 (2013).

55. He, X. Y. et al. Gambogic acid induces EGFR degradation and Akt/mTORC1 inhibition through AMPK dependent-LRIG1 upregulation in cultured U87 glioma cells. Biochem. Biophys. Res Commun. 435, 397-402 (2013).

56. Komatsu, M. \& Ichimura, Y. Physiological significance of selective degradation of p62 by autophagy. FEBS Lett. 584, 1374-1378 (2010).

57. Chen, L. et al. AMPK activation by GSK621 inhibits human melanoma cells in vitro and in vivo. Biochem. Biophys. Res Commun. 480, 515-521 (2016).

58. Liu, W. et al. Targeted activation of AMPK by GSK621 ameliorates H2O2induced damages in osteoblasts. Oncotarget 8, 10543-10552 (2017).
59. Wu, Y. H., Li, Q., Li, P. \& Liu, B. GSK621 activates AMPK signaling to inhibit LPSinduced TNFalpha production. Biochem. Biophys. Res Commun. 480, 289-295 (2016).

60. Neal, R. D., Hamilton, W. \& Rogers, T. K. Lung cancer. BMJ 349, g6560 (2014).

61. Rosell, R. \& Karachaliou, N. Lung cancer in 2014: optimizing lung cancer treatment approaches. Nat. Rev. Clin. Oncol. 12, 75-76 (2015).

62. Keith, R. L. \& Miller, Y. E. Lung cancer chemoprevention: current status and future prospects. Nat. Rev. Clin. Oncol. 10, 334-343 (2013).

63. Raez, L. E. \& Lilenbaum, R. Chemotherapy for advanced non-small-cell lung cancer. Clin. Adv. Hematol. Oncol. 2, 173-178 (2004).

64. Langer, C. \& Lilenbaum, R. Role of chemotherapy in patients with poor performance status and advanced non-small cell lung cancer. Semin. Oncol. 31 8-15 (2004).

65. Liao, X. Z. et al. Cordycepin reverses cisplatin resistance in non-small cell lung cancer by activating AMPK and inhibiting AKT signaling pathway. Front Cell Dev. Biol. 8, 609285 (2020).

66. Wu, Y. et al. Polyphyllin I activates AMPK to suppress the growth of non-smallcell lung cancer via induction of autophagy. Arch. Biochem. Biophys. 687, 108285 (2020).

67. You, J., Cheng, J., Yu, B., Duan, C. \& Peng, J. Baicalin, a Chinese herbal medicine, Inhibits the proliferation and migration of human non-small cell lung carcinoma (NSCLC) cells, A549 and H1299, by activating the SIRT1/AMPK signaling pathway. Med Sci. Monit. 24, 2126-2133 (2018).

68. Luo, Z. et al. Metformin induces apoptotic cytotoxicity depending on AMPK PKAVGSK-3beta-mediated c-FLIPL degradation in non-small cell lung cancer. Cancer Manag Res. 11, 681-689 (2019).

69. Heavey, S., O'Byrne, K. J. \& Gately, K. Strategies for co-targeting the PI3K/AKT/ mTOR pathway in NSCLC. Cancer Treat. Rev. 40, 445-456 (2014).

70. Tan, A. C. Targeting the PI3K/Akt/mTOR pathway in non-small cell lung cancer (NSCLC). Thorac. Cancer 11, 511-518 (2020).

71. Wang, S. et al. SH2B1 promotes NSCLC cell proliferation through PI3K/Akt/ mTOR signaling cascade. Cancer Cell Int. 18, 132 (2018).

72. Kumar, V. B. et al. Antroquinonol inhibits NSCLC proliferation by altering PI3K mTOR proteins and miRNA expression profiles. Mutat. Res. 707, 42-52 (2011).

73. Saxton, R. A. \& Sabatini, D. M. mTOR signaling in growth, metabolism, and disease. Cell 168, 960-976 (2017).

74. Laplante, M. \& Sabatini, D. M. mTOR signaling in growth control and disease. Cell 149, 274-293 (2012)

75. Guertin, D. A. \& Sabatini, D. M. Defining the role of mTOR in cancer. Cancer Cell. $12,9-22$ (2007)

76. Gwinn, D. M. et al. AMPK phosphorylation of raptor mediates a metabolic checkpoint. Mol. Cell. 30, 214-226 (2008).

77. Liu, G. et al. Role of autophagy and apoptosis in non-small-cell lung cancer. Int J. Mol. Sci. 18, (2017).

78. Zhang, M., Su, L., Xiao, Z., Liu, X. \& Liu, X. Methyl jasmonate induces apoptosis and pro-apoptotic autophagy via the ROS pathway in human non-small cell lung cancer. Am. J. Cancer Res. 6, 187-199 (2016).

79. Khan, S. H. \& Kumar, R. Role of an intrinsically disordered conformation in AMPK-mediated phosphorylation of ULK1 and regulation of autophagy. Mol. Biosyst. 8, 91-96 (2012).

80. Jang, M. et al. AMPK contributes to autophagosome maturation and lysosomal fusion. Sci. Rep. 8, 12637 (2018). 